\title{
Application of Artificial Neural Networks in Autonomous Mission Planning for Planetary Rovers
}

\section{Zhaoyu Li $\mathbf{i}^{\mathbf{1}}$}

Institute of Deep Space Exploration Technology, School of Aerospace Engineering, Beijing Institute of Technology,

Beijing, China 100081

\section{Rui $\mathbf{X} \mathbf{u}^{2}$}

Institute of Deep Space Exploration Technology, School of Aerospace Engineering, Beijing Institute of Technology,

Beijing, China 100081

E-mail:xuruiebit.edu.cn

\section{Yanhan $\mathrm{Ma}^{3}$}

Shanghai Institute of Satellite Engineering,

Shanghai, China 200240

The mission planning is a field of Artificial Intelligence (AI), which is intractable in the general case. The mission planning problems are usually complex and difficult to be solved by conventional techniques. Practically, researchers turn to solve these problems which are limited by deterministic parameters with all kinds of heuristic algorithms. As to the problems in real world, these approaches are defective sometimes because of complex constraints. In this study, artificial neural networks (ANN) are applied to the mission planning. The utilization of artificial neural networks on solving planning problems is also examined; at the same time, an ANN model is developed by using multiple layer perceptron networks and carried out to solve a real world problem in rover domain.

CENet2015

12-13 September 2015

Shanghai, China

\footnotetext{
${ }^{1}$ Speaker

${ }^{2}$ Corresponding Author

${ }^{3}$ The authors gratefully acknowledge the support of the Civil Aerospace Research Project of China.
} 


\section{Introduction}

Autonomous planning is an abstract and well-thought reasoning process in terms of actions. This process chooses and organizes a set of actions through predicting action effects in order to achieve some given objects as well as possible [1]. The mission planning problems are a kind of planning problems which have been proved NP-hard by Bernhard Nebel [2]. Usually, these problems are complex and difficult to be solved by conventional techniques due to highly complex constraints [3].

As some planetary rovers are usually on planets far from the earth, such as Mars, it leads to long time to communicate between the rover and the ground station; therefore, it is necessary to use autonomous mission planning for planetary rovers. The rover domain is constructed as a simplified representation of the problem that simulates the NASA Mars Exploration Rover missions [4]. The rover domain involves planning for several rovers, equipped with different sets of equipment to traverse a planet surface. As to this real problem, it is difficult to search an efficient solution by using traditional approaches which often consider small-sized problems with deterministic parameters.

Practically, researchers turn to search its near-optimal solutions with all kinds of heuristic algorithms [5], for example, the Extensible Universal Remote Operations Planning Architecture (EUROPA) and the SPIKE system for intelligent scheduling of Hubble Space Tele-scope; however, the heuristic algorithms have common disadvantages of symbolic artificial intelligence, for example, the researchers should know the characteristics of the system and then adopt appropriate evaluation function. As a result, in this study, we use artificial neural networks (ANNs) to seek the solution of mission planning problems in the rover domain.

Several researches using ANNs have been done in terms of the motion planning, which is in a lower level relative to mission planning in the planning field. Youssef Bassil proposed an ANN model made out of three layers and trained in offline mode using back-propagation supervised learning algorithm [6]. Yitong Zhang used the Hopfield neural network for solving the vehicle routing problem of "advanced request" to shorten the delivery path length and reduce the logistics [7]. Malek Alaeddin solved the optimization problems in path planning of planetary rovers with specific recurrent neural network [8]. These researches provide reference for applying ANNs on the mission planning.

In this paper, we propose an algorithm based on the multiple layer perceptron (MLP) to select actions in planning process. At last, the method based on MLP is carried out to solve an application problem in the rover domain.

\section{Autonomous Mission Planning Problem Formulation}

The formulation of the planning problems should include states, the action set, statetransition relations, and a mission. Here, we adopt the representation for classical planning to describe the domain.

The restricted state-transition system is represented by a triple

$$
\Sigma=(S, A, \gamma)
$$

Where $S$ is a set of states, $A$ is a set of actions and $\gamma$ is a state-transition function which is used to describe the state-transition relationship.

The planning problem of the rover is a triple

$$
\mathrm{P}=\left(\Sigma, S_{0}, g\right)
$$

Where is the state-transition system as mentioned above, $s_{0}$ is the initial state which is a member of $S$, and $g$ is the target state and also one member of $S$. The state-transition system is independent of the goal and the initial state.

Based on the definition above, the input of the ANN can be represented by

$$
\text { Input }=\left[s_{\text {now }}, g\right]
$$


$s_{\text {now }}$ is the current states, and $s_{\text {now }}=s_{0}$ at the first time of using ANNs.

The output of the ANN is the next set of states that relevant systems of the planning object (e.g. the rovers) should be in. In equation (4), $m$ indicates that there are $m$ systems in the planning object.

$$
\text { Output }=\left[s_{1}, s_{2}, \ldots, s_{m}\right]
$$

\section{Artificial Neural Networks Based Planning Algorithm}

As one of the main approaches to achieve the artificial intelligence, the artificial neural networks (ANNs) are popular in many areas and provide a novel approach for fast mission planning because of storage of knowledge, parallel computing, as well as ability to learn and solve complicated problems. ANNs can also find solutions for problems with unknown or complex internal relations. If some information is ignored at the moment of establishing the model, ANNs can make up during learning. On the basis of the ANN model having been used in planning and scheduling fields, a multilayer perceptron (MLP) network model with variable learning rate back propagation algorithm is used in this paper.

\subsection{ANNs Model}

The proposed network consists of three layers: input layer, one hidden layer, and output layers. Fig. 1 shows the structure of the proposed MLP network for the autonomous mission planning system. The structure emphasizes the importance of the appointed task which determines the systems that need to perform actions and the constraints among these systems.

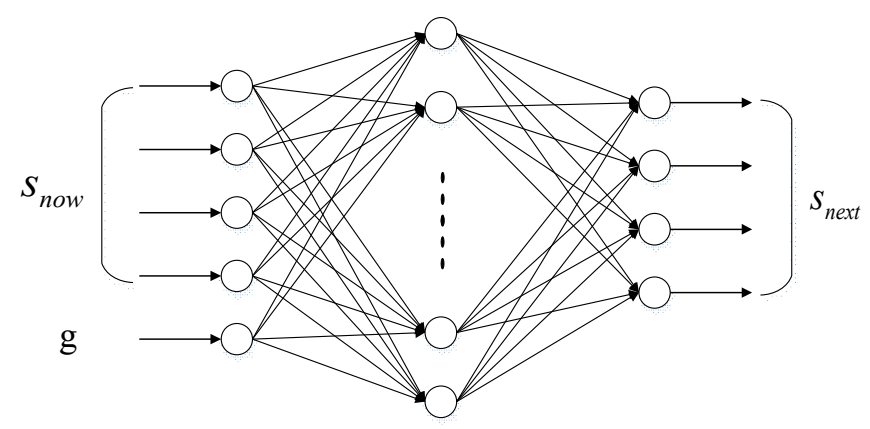

Figure 1: Structure of the Used MLP Network

The input of network includes an initial state $s_{0}$ and a target state $g$, while the output is the next state set. Here, $P_{0}$ represents the initial mission planning problem; so the input of MLP can be expressed by

$$
P_{0}=\left[s_{0}, g\right]
$$

Where $s_{0}=\left[s_{0}^{1}, s_{0}^{2}, s_{0}^{3}, s_{0}^{4}\right], s_{0}^{1}$ represents the initial state of the first system of the rover, similarly $s_{0}^{2}, s_{0}^{3}, s_{0}^{4}$.

The output is the next state sequence. The MLP is responsible for finding the next state of each system of the rover according to the initial state.

Because of different target states, the systems that need to change their states are different. Some systems need to change with others not. The ANN model adjusts the weight in each layer to decide which systems are involved.

\subsection{ANNs Based Algorithm}

The autonomous mission planning approach as we adopt is the forward searching in the state space. Using MLP network selects actions in the course of searc, the input signal of the MLP propagates through the network in a forward direction and on a layer-by-layer basis. 
According to a forward-search planning algorithm in state-space planning, we design a planning algorithm based on the MLP model in Fig. 2 [4].

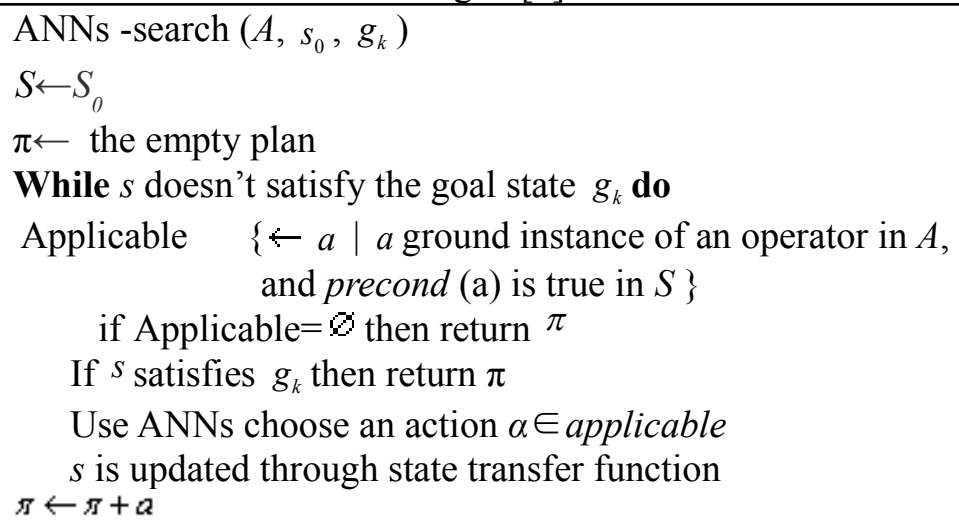

Figure 2: Autonomous Mission Planning Searching Algorithm Based on the MLP Network

Designing the MLP neural network-based planning algorithm to choose the better action sequence constantly and then drive the planning object to get to the target state by executing as few actions as possible. If not using MLP, the mission planning system will choose actions tentatively. When the mission planning system cannot reach the target state, it will re-plan from the initial state which will waste lots of time. Utilizing MLP can find a better planning solution.

\section{Simulation and Result Comparison}

In this section, we take the rover domain as an example to verify the mission planning method based on ANNs.

\subsection{Data Encoding}

The input data set should include the mission, loading states, the position, camera statesand communication states of the rover. The output plan provides the next state of each system of the rover (include loading system, camera system and communication system) and the position the rover need to stay or move to. Details are as follows:

Input: $I_{i}=\left(g, l_{i}, \operatorname{pos}_{i}, c a_{i}, c o m_{i}\right)$, where $i$ represents the $i^{\text {th }}$ input. $g$ is the mission, $l_{i}$ is the loading state, $\operatorname{pos}_{i}$ is the position, $c a_{i}$ is the camera state, and $c{ } m_{i}$ is the communication state. Specific encoding is shown in Table 1.

\begin{tabular}{llll}
\hline Loading states & Camera states & Communication states & Rover position \\
\hline 1 (loading) & 1 (take photo) & 1 (communicate) & 1 (initial) \\
2 (working) & 2 (idle) & 2 (idle) & 2 (moving) \\
3 (analyzing) & & & 3 (goal) \\
4 (transfer) & & & \\
5 (unloading) & & & \\
\hline
\end{tabular}

Table 1: Encoding for All Systems

Output: $O=\left(a_{l}, a_{p o s}, a_{c a}, a_{c o m}\right)$, where $a_{l}$ represents the next loading state or action. $a_{p o s}$ represents where to go next. $a_{c a}$ is the next camera state; and $a_{c o m}$ is the next communication state.

\subsection{Simulation}

The data used in this study is gained from a rover simulation system. The ANN is trained by fifty data sets. The learning curve is shown in Fig. 3. The best training error is 0.006 . 


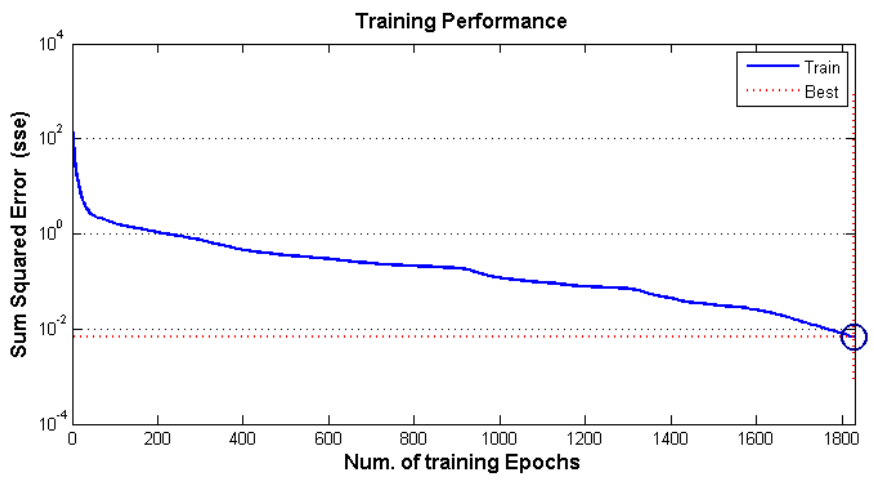

Figure 3: Learning Curve of the ANN

Here, one mission not included in the training data is given to verity the availability of ANN. Initial states and goal states are shown in Table 2. This mission means the rover has to move from the initial position to the goal position, and take one photo of the target at the goal position.

\begin{tabular}{lll}
\hline & Initial states & Goal states \\
\hline Loading & 5 & 5 \\
Camera & 2 & 1 \\
Communication & 2 & 2 \\
Position & 1 & 3 \\
\hline
\end{tabular}

Table 2: Initial States and Goal States for Simulation

Fig. 4 shows the planning result. The number in boxes is the encoding of relative systems in Table 1.

\begin{tabular}{|c|c|c|c|c|}
\hline \multirow[b]{2}{*}{ Loading states } & Initial states & \multicolumn{2}{|c|}{ Procedures } & \multirow{2}{*}{$\begin{array}{c}\text { Goal states } \\
5 \\
\end{array}$} \\
\hline & 5 & 5 & 5 & \\
\hline Camera states & 2 & 2 & 1 & 2 \\
\hline Communication states & 2 & 2 & 2 & 2 \\
\hline Rover position & 1 & 2 & 3 & 3 \\
\hline
\end{tabular}

Figure 4: The Planning Result

In order to compare the run times of ANN with that of a heuristic algorithm based on a cost of the transition between actions, six missions are given. Five missions thereof have different initial states and goal states. The run times are shown in Table 3, which indicates that ANN algorithm is faster to solve mission planning problems for planetary rovers.

\begin{tabular}{lll}
\hline Mission & Run times (s) & \\
\hline & ANN & Heuristic \\
\hline g1 & 0.024 & 0.835 \\
g2 & 0.015 & 0.643 \\
g3 & 0.027 & 0.98 \\
g4 & 0.019 & 0.560 \\
g5 & 0.020 & 0.843 \\
g6 & 0.029 & 0.918 \\
\hline
\end{tabular}

Table 3: Comparison of Run Times of ANN Algorithm with A Heuristic Algorithm

\section{Conclusion}

In this paper, the usage of ANNs is examined to realize the mission planning. ANNs are used in a planning process to choose the next state set according to initial states.

The results obtained from the network are an optimal order for planning mission. The multiple layer perceptron neural networks are used and the weights for each layer are obtained by the least square method in this study. There is a mission planning problem in rover domain 
with 4 systems to find the optimal processing orders. The simulation results show that ANNs can be used in mission planning field and can find plans faster.

As a further work, the input applied for the neural network can be expanded for more detailed planning procedures. For example, some additional input can be used as specific positions, time of each action, targets the camera takes photos of, etc. More details are considered; it will be more close to apply ANNs on real mission planning problems.

\section{References}

[1] M. Ghallab, D. Nau, P. Traverso. Automated planning: theory and practice [M]. Elsevier, San Francisco, CA. pp, 1-13 (2004).

[2] B. Nebel. On the computational complexity of temporal project, planning, and plan validation [J]. Artificial Intelligence. 66 (1), 125-160 (1994).

[3] N. Muscettola, P. Nayak, B. Pell, and B. Williams. Remote Agent: to boldly go where no AI system has gone before [J]. Artificial Intelligence 103(1-2), 5-47 (1998).

[4] N. Meuleau, E. Benazera, R. I. Brafman, and E. A. Hansen. A heuristic search approach to planning with continuous resources in stochastic domains [J]. Journal of Artificial Intelligence Research. 34, 27-59 (2009).

[5] A. F. Guneri, A .T. Gumus. The usage of artificial neural networks for finite capacity planning [J]. International Journal of Industrial Engineering. 15(1), 16-25 (2008).

[6] Y. Bassil. Neural network model for path-planning of robotic rover systems [J]. International Journal of Science and Technology. 2(2), 94-100 (2012).

[7] Y. Zhang, G. Zhao. Research on multi-service demand path planning based on continuous Hopfield neural network [J]. Lecture Notes in Electrical Engineering, 286, 417-430 (2015).

[8] A. Malek., L. J K. Abad, S. Khodayari-Samghabadi. Semi-infinite programming to solve armed robot trajectory problem using recurrent neural network [J]. International Journal of Robotics \& Automation. 30(2), 113-118 (2015). 